\title{
Impacto de la regla de decisión en el modelado de la difusión de innovaciones
}

\author{
Lorena Cadavid ${ }^{\mathrm{a} *}$, Carlos Jaime Franco Cardona ${ }^{\mathrm{a}}$ \\ a*Universidad Nacional de Colombia, Medellín, Colombia, dlcadavi@unal.edu.co
}

\begin{abstract}
Resumen
Este artículo analiza el impacto de la regla de decisión que representa el comportamiento de los individuos en la curva de difusión pronosticada por los modelos de difusión de innovaciones a nivel individual. Para ello, se hace uso de un modelo basado en agentes, en el cual la difusión ocurre dentro de una red tipo mundo pequeño, y se analiza el fenómeno usando 4 reglas de decisión diferentes: (1) una regla de umbrales con externalidades positivas, (2) una regla de umbrales con externalidades positivas y negativas, (3) una regla basada en el modelo de Bass y (4) una regla basada en la Teoría del Comportamiento Planeado. Los resultados obtenidos rechazan la hipótesis de igualdad entre las diferentes curvas de difusión. Se concluye que la regla de decisión tiene un impacto significativo en la curva de difusión pronosticada por los modelos de difusión a nivel individual.
\end{abstract}

\section{Palabras clave}

Simulación. Difusión de innovaciones. Adopción de innovaciones. Modelado basado en agentes. Regla de decisión.

\section{Introducción}

La representación matemática o computacional del proceso de la difusión de una innovación se conoce como modelado de la difusión de innovaciones, y ha sido un tópico de importancia académica y práctica desde la década del 60, cuando aparecieron los primeros trabajos (Bass, 1969; Mahajan et al., 1990). Los estudios en el campo buscan explicar la propagación de una innovación desde la perspectiva de las interacciones entre los consumidores y las comunicaciones (Peres et al., 2010; Rao \& Kishore, 2010), así como lograr el entendimiento de la influencia de factores particulares detrás de los patrones de difusión observados para predecir la difusión de una innovación (Montalvo \& Kemp, 2008).

La metodología de modelado a nivel individual lidera las investigaciones actuales en el modelado de la difusión de innovaciones (Kiesling et al., 2012). Los modelos basados en esta metodología tienen dos componentes fundamentales: (1) modelado de la red de interacción que afecta la elección de la adopción y (2) modelado de la regla de decisión o mecanismo para determinar cuándo la adopción de la innovación tiene lugar para cada individuo (Georgescu \& Okuda, 2008; Kiesling et al., 2012).
Atendiendo a diversos argumentos, cada estudio selecciona diferentes técnicas de modelado tanto para la red de interacción como para la regla de decisión, y no existe una uniformidad en estas técnicas de modelado a lo largo de las investigaciones. Este hecho armoniza con la afirmación de Kiesling et al. (2012), según la cual los modelos de difusión a nivel individual están en un estado temprano de desarrollo, que se evidencia, entre otros hechos, en la falta de estándares de modelado de la técnica.

El objetivo de este artículo es analizar el impacto que tiene la regla de decisión que representa el comportamiento de los individuos en la curva de difusión pronosticada por los modelos de difusión de innovaciones a nivel individual. Para ello, se hace uso de un modelo basado en agentes, en el cual la difusión tiene lugar dentro de una red que exhibe características de mundo pequeño. El fenómeno de la difusión ocurre bajo 4 escenarios diferentes, cada uno de los cuales usa una de 4 posibles reglas de decisión, en las cuales la decisión de adopción depende: (1) favorablemente de la cantidad de vecinos adoptadores; (2) tanto favorable como desfavorablemente de la cantidad de vecinos adoptadores; (3) de los vecinos 
adoptadores, de la cantidad de adoptadores de la red y de la propensión a adoptar; y (4) de la actitud hacia la adopción, de la norma subjetiva y de la percepción de control (siguiendo los lineamientos de la Teoría del Comportamiento Planeado (Ajzen, 1985)). Los resultados obtenidos rechazan la hipótesis de igualdad entre las diferentes curvas de difusión, así que es posible concluir que la regla de decisión tiene un impacto significativo en la curva de difusión pronosticada por los modelos de difusión a nivel individual tanto en forma como en velocidad. Dado que diferentes reglas arrojan diferentes patrones de difusión, se recomienda el desarrollo de metodologías de validación de las reglas de decisión empleadas en los modelos de difusión a nivel individual.

Los resultados contribuyen a la madurez de los modelos de difusión a nivel individual y a la aceleración de su eventual contribución en el entendimiento y la predicción del fenómeno de la difusión de las innovaciones.

\section{Modelos de difusión a nivel individual}

De acuerdo con Gilbert (1997), el modelado a nivel individual es la técnica de modelado más usada para la simulación en las ciencias sociales. En los últimos años, los $\mathrm{MDl}$ se han usado cada vez con más frecuencia en el campo de la difusión de innovaciones, posicionándose como una técnica prometedora en las investigaciones que lideran el tema (Goldenberg et al., 2000). De acuerdo con Rahmandad \& Sterman (2008), esta técnica resulta natural para modelar procesos de difusión, no sólo porque las dinámicas de contagio involucran características importantes de sistemas complejos (como realimentación positiva y negativa, retardos, no linealidad en las variables, eventos estocásticos y heterogeneidad individual), sino porque la topología de la red es especialmente importante en el proceso de difusión.

En los MDl el elemento atómico del modelo es la unidad de análisis, que usualmente es un individuo, un hogar o una organización (Manson, 2006). La heterogeneidad de los consumidores, sus interacciones sociales y sus procesos de toma de decisiones pueden ser modelados explícitamente; de este modo, las dinámicas a nivel macro del sistema social emergen dinámicamente desde el comportamiento individual agregado y las interacciones entre los agentes (Kiesling et al., 2012), de manera similar a la sugerida por Schelling (1978).

Los MDI se componen de dos elementos principales o módulos de modelado: (1) el modelado de la interacción social entre las unidades de análisis, y
(2) el modelado del proceso de la toma de decisiones de dichas unidades

La interacción o influencia social es un concepto que hace referencia a la influencia transmitida a través de vínculos de comunicación directos o indirectos entre los individuos (como en el boca a boca); (2) meso, en el cual se encuentran todas las influencias que surgen colectivamente del ambiente social inmediato de un individuo (es decir, su vecindario en la red social); y (3) macro, el cual hace referencia a las interacciones a nivel de la sociedad como un todo (Kiesling et al., 2012). El modelado de la toma de decisiones, por su parte, permite que las decisiones de los individuos o unidades de análisis sean explícitamente incorporadas en los modelos de difusión (Kemp \& Volpi, 2008). Las secciones 2.1 y 2.2 ampliarán cada uno de estos módulos de modelado.

\subsection{Modelado de la interacción social}

La estructura social en la cual un individuo se encuentra inmerso es el primero de los elementos necesarios para el modelado de la difusión de las innovaciones a nivel individual. Este hecho es bien sabido desde antes de que los MDl tuvieran lugar; en efecto, como sostiene Rogers (1983), la difusión de una innovación no puede ser explicada únicamente como el resultado de la heterogeneidad entre los individuos, sino fundamentalmente como un proceso social, de manera que ésta última se constituye como el sustrato dentro del cual una innovación se propaga (Macvaugh \& Schiavone, 2010).

La red afecta la difusión, no solamente a través de las comunicaciones boca a boca, sino a través de las externalidades de red. Las externalidades de red tienen lugar cuando la utilidad que obtiene un individuo al adoptar una innovación cambia en función de la cantidad de individuos que previamente adoptaron la innovación (Katz \& Shapiro, 1986; Rohlfs, 2001); las externalidades pueden ser globales, cuando el consumidor toma en cuenta el sistema social completo al evaluar la utilidad del producto en términos del número de adoptadores, o locales, cuando la utilidad el producto sólo considera la adopción en la red social más cercana (Katz \& Shapiro, 1992).

La tipología de la red social resulta crucial para el análisis de la difusión de innovaciones. En este sentido, se considera que las redes que mejor se ajustan a la representación de las redes sociales son las llamadas redes mundo pequeño (small-world) (Watts, 2000), un intermedio entre las redes aleatorias y las redes completamente conectadas, que exhiben un diámetro pequeño y una alta agrupación (Barabási, 1999a, b). 
Numerosos estudios en el modelado de la difusión de innovaciones en los últimos años exploran el impacto de la estructura de la red en la difusión de una innovación, comparando diferentes estructuras bajo una misma regla de decisión. En general, los resultados obtenidos en estos estudios depende del tipo de supuestos que se hicieron sobre el proceso de toma de decisiones de los individuos, de manera que muchos de los resultados son contradictorios entre sí (Kiesling et al., 2012). La sección 3.2 explora el modelado de la toma de decisiones en el campo de la difusión de innovaciones, el segundo componente de los MDI.

\subsection{Modelado de la toma de la decisión}

Un elemento clave en los modelos de difusión basados en interacciones individuales es la representación explícita del proceso de toma de decisiones de los individuos, especialmente relativo a la decisión de adoptar o no una innovación. Este elemento conduce al campo de la adopción de las innovaciones, el cual busca explicar el proceso a través del cual un individuo toma la decisión de adoptar o no la innovación (Montalvo \& Kemp, 2008; Venkatesh $\&$ Bala, 2008; Chuttur, 2009).

El estudio de cuáles factores conducen la adopción tecnológica es amplio y multidisciplinario, pues un gran número de condiciones internas (como las limitaciones personales de los adoptadores potenciales) y externas (como canales de comunicación ineficaces) pueden inhibir el éxito del proceso de adopción (Rogers, 1983; Bagozzi, 2007; Macvaugh \& Schiavone, 2010).

En un esfuerzo por hacer evidente el potencial del modelado a nivel individual de la difusión de innovaciones, Kiesling et al. (2012) encuentran que los modelos de adopción usados en los MDI corresponden a reglas que pueden agruparse en una de cinco categorías. Las más simples de ellas corresponden a las reglas de umbrales, mientras que las más complejas hacen referencia a las aproximaciones psicosociológicas, como se expondrá a continuación

\subsubsection{Reglas de umbrales}

En este tipo de reglas, las cuales son de gran uso en los MDI (Georgescu \& Okuda, 2008), los individuos pueden adoptar una innovación a través de dos vías: (1) una auto-convicción sobre la conveniencia de la adopción, y (2) una convicción formada después de la interacción con otros individuos (Goldenberg \& Efroni, 2001).

La primera vía es la que recorren los individuos clasificados como innovadores por Rogers (1983, p.
247), los cuales representan una pequeña fracción de la población. Los innovadores son individuos que tienen actitudes hacia la innovación y hacia el riesgo diferentes de las de los demás adoptadores, siguiendo un proceso de decisión independiente de las dinámicas de red (Kemp \& Volpi, 2008). La segunda vía es la recorrida por los demás adoptadores en la población; para ellos, la decisión de adopción tiene lugar cuando un porcentaje de individuos cercanos adopta la innovación, y dicho porcentaje o umbral de exposición puede ser fijo o variable a lo largo de la población. La decisión puede ser determinística si el individuo adopta una vez dicho umbral es superado, o probabilística si el individuo adopta con una cierta probabilidad una vez el umbral es superado (Kiesling et al., 2012).

Sin embargo, los estudios empíricos revelan que bajo determinadas circunstancias los individuos pueden encontrarse en una situación de sobre-exposición a la innovación cuando la cantidad de vecinos adoptadores es muy alta, lo cual disminuye la atractividad de la adopción dado que la innovación deja de estar de moda (externalidad negativa) (Buchanan, 1969; Allen, 1982; Katz \& Shapiro, 1986).

Este hecho representa un gran desafío para los modeladores de los procesos de difusión, que consiste en la separación de los distintos tipos de efectos interpersonales en diferentes parámetros, Aunque pocas investigaciones exploran esta idea, el trabajo de Alkemade \& Castaldi (2005) es una buena representación de ella. La conclusión general de estos estudios es que los efectos de este tipo de externalidades son, por lo menos, tan fuertes como los de las externalidades positivas, y que pueden inhibir el proceso de la difusión desestimulando la adopción de los individuos (Peres et al., 2010).

\subsubsection{Aproximaciones psicosociológicas}

Las aproximaciones psicosociológicas se consideran las más sofisticadas y menos parsimoniosas (Kiesling et al., 2012), y se basan en las teorías psicológicas y sicológicas sobre el comportamiento. Comúnmente estas aproximaciones hacen uso del modelo de Ajzen (1985) conocido como la Teoría del Comportamiento Planeado (Theory of Planned Behavior, en adelante TPB). La TPB sugiere que el comportamiento de un individuo está determinado por su intención previa a adoptar dicho comportamiento, y que dicha intención está determinada, a su vez, por tres factores: (1) la actitud o creencias que tiene el individuo sobre el comportamiento y la evaluación de los resultados esperados al desempeñarlo; (2) las normas subjetivas o lo que el individuo cree que otros individuos importantes para él pensarían si él 
desempeñara el comportamiento (Ellis \& Fisher 1994, apud Hsu \& Lin, 2008); y (3) el control comportamental percibido o qué tan bien cree el individuo poder desempeñar el comportamiento (Hale et al., 2002).

De esta forma, la intención de desempeñar el comportamiento de un individuo será una suma de la medida de actitud, normas subjetivas y control percibido, ponderada por unos pesos que se hallan de forma empírica (Hale et al., 2002).

Este tipo de reglas fortalece el concepto de heterogeneidad de los individuos. En términos generales, la heterogeneidad de la población hace alusión a variedad en el grado de innovación de los consumidores, la sensibilidad al precio y las necesidades, entre otras variables; estas diferencias permiten que el producto tenga lugar en el mercado, y la distribución de las mismas en un sistema social específico determina la forma del patrón de distribución (Kemp \& Volpi, 2008; Peres et al., 2010).

La heterogeneidad en la propensión a adoptar es la aproximación más común para incorporar la heterogeneidad de los individuos en los MDI (Kiesling et al., 2012). La heterogeneidad en los precios de reserva (Varian, 1985) es un enfoque similar al anterior, en el cual el factor a variar es el precio de reserva del individuo (precio por debajo del cual un individuo adopta la innovación).

Si bien diversas investigaciones exploran el impacto de diferentes estructuras de la red en la difusión de una innovación, el trabajo que se ha adelantado en la evaluación del impacto de la regla de decisión en la velocidad o el patrón de la difusión de las innovaciones es poco.

\section{Metodología}

Se desarrolló un modelo basado en agentes para representar el fenómeno de la difusión de una innovación. Esta técnica ha sido ampliamente usada en la representación del fenómeno de la difusión de una innovación en los últimos años, especialmente por su poder explicativo para la construcción de teoría y para el análisis de políticas (Berger, 2001; Kiesling et al., 2012).

En el modelo desarrollado, los individuos son considerados agentes que toman decisiones en función de una regla que opera del mismo modo para todos, pero que es alimentada por insumos diferentes en función de la estructura de la red y de la heterogeneidad de los individuos. La estructura de la red representada exhibe características de mundo pequeño, y fue implementada haciendo uso del algoritmo generativo desarrollado por Watts \& Strogatz (1998), y la heterogeneidad de los individuos está vinculada con la regla de decisión usada. En cada paso de simulación, los individuos deciden adoptar o no la innovación en función de una regla de decisión implementada; cada simulación comprende un total de 20 pasos.

El modelo considera los dos tipos de reglas de decisión enunciados por Kiesling et al. (2012): (1) regla de umbrales con externalidades positivas y (2) una regla sociológica. Además, se diseñaron otras dos reglas intermedias: (3) regla de umbrales con externalidades positivas y negativas, y (4) regla basada en el modelo de Bass. A continuación se explica el funcionamiento de cada regla.

R1: Regla de umbrales con externalidades positivas: esta regla se modeló considerando un porcentaje inicial de innovadores y un umbral individual después del cual la adopción ocurre, como se presenta en la Figura 1.

Después de configurar de manera aleatoria una cantidad determinada de adoptadores iniciales al comenzar la simulación, en cada paso de simulación se calcula, para cada individuo no adoptador, el porcentaje de vecinos inmediatos (aquellos con los que tiene vínculo directo) que ha adoptado la innovación respecto al total de vecinos inmediatos del mismo individuo. Si este porcentaje supera el umbral de adopción del individuo, el individuo se convierte en adoptador de la innovación. Debe tenerse presente que, si bien el porcentaje de adoptadores iniciales es fijo a lo largo de la difusión, la elección de cuáles individuos componen dicho porcentaje es aleatoria.

A fin de hacer de esta regla la más simple posible, el umbral de adopción se determinó igual para todos los individuos de la red social, de manera que la heterogeneidad de la población depende de forma exclusiva de la posición que tiene cada individuo dentro de la red social en la cual la difusión ocurre. Asimismo, la regla se modeló de manera determinística, es decir que la adopción siempre tendrá lugar una vez el porcentaje de vecinos adoptadores de un individuo supera el umbral de adopción.

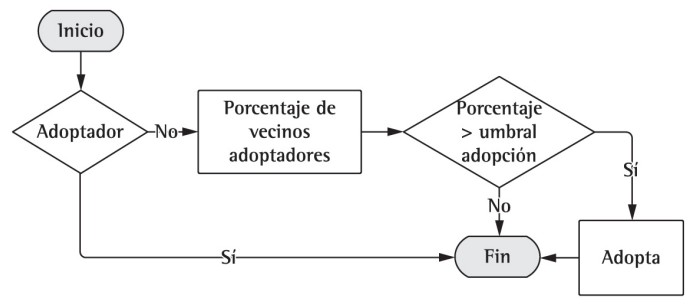

Figura 1. Regla de umbrales con externalidades positivas. Fuente: los autores. 
R2: Regla de umbrales con externalidades positivas y negativas: esta regla recoge la descripción teórica de los conceptos de exposición y sobre-exposición a una innovación, modelándolos de forma similar a las lógicas subyacentes en el juego de la vida propuesto por Jhon Conway y publicado por Gardner (1970). En este sentido, adicional al umbral de adopción similar al considerado en la regla de umbrales con externalidades positivas, se incluyó un umbral de abandono (superior al umbral de adopción) por encima del cual el adoptador abandona la innovación, como se presenta en la Figura 2.

De esta manera, en cada paso de la simulación un individuo no adoptador puede convertirse en adoptador si el porcentaje de vecinos adoptadores supera su umbral de adopción, y un individuo adoptador puede abandonar la adopción si el porcentaje de vecinos adoptadores supera su umbral de abandono.

Tanto el umbral de adopción como el umbral de abandono se consideraron iguales para todos los individuos de la red social. Al igual que en la regla con externalidades positivas, si bien el porcentaje de innovadores es fijo a lo largo de la difusión, la elección de cuáles individuos componen dicho porcentaje es aleatoria.

R3: Regla de Bass: aunque el modelo de Bass pertenece a la categoría del modelado a nivel agregado, de desarrolló una regla de decisión con base en los conceptos recogidos por este modelo que se presenta en la Figura 3.

En cada paso de simulación, los individuos no adoptadores cambian su umbral de adopción y calculan el valor de la regla de decisión de Bass. $\mathrm{Si}$ este valor supera dicho umbral, el individuo se convierte en adoptador.

El valor de la regla de Bass para cada individuo en cada paso de simulación está determinado por la Ecuación 1.

Bass $_{i t}=\frac{P+I \times \frac{A_{t}}{N}+E \times \frac{V A_{i t}}{V_{i}}}{3}$

\section{Donde:}

- Bass $_{\mathrm{it}}=$ valor de la regla de Bass para el individuo $i$ en el momento $t$

- $\mathrm{P}=$ propensión a la innovación

- 1 = coeficiente de imitación

- $A_{t}=$ cantidad de adoptadores que hay en la red en el momento $t$.

- $\mathrm{N}=$ total de individuos en la red

- $\mathrm{E}=$ coeficiente de externalidad

- $\mathrm{VA}_{\mathrm{it}}=$ cantidad de vecinos adoptadores al individuo $i$ en el momento $t$.

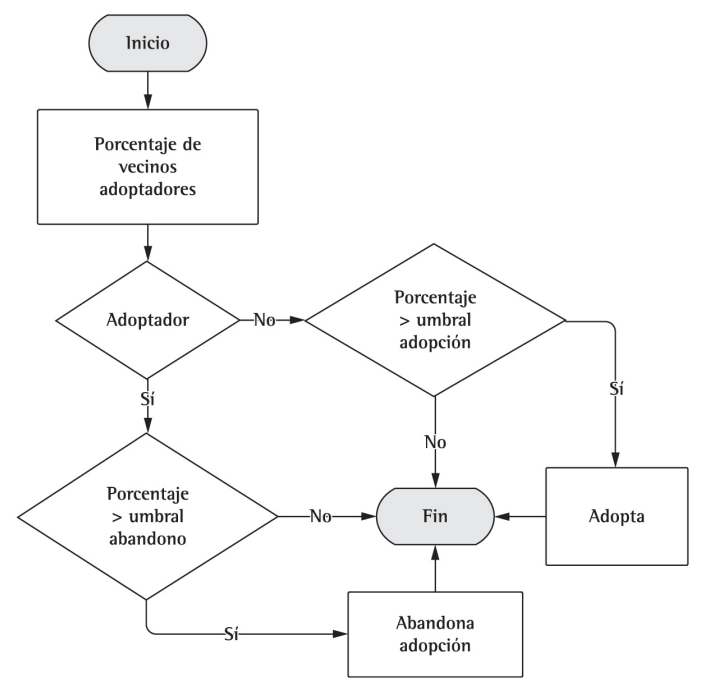

Figura 2. Regla de umbrales con externalidades positivas. Fuente: los autores.

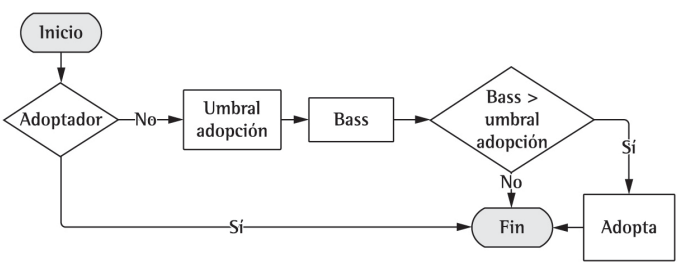

Figura 3. Regla de Bass. Fuente: los autores.

- $\mathrm{V}_{\mathrm{i}}=$ cantidad de vecinos al individuo $i$.

De acuerdo con esta regla, la decisión de adopción del individuo depende de la propensión a la innovación propia en el individuo (la cual es detonada con la publicidad que recibe sobre la innovación, similar a una influencia externa), y de la cantidad de vecinos adoptadores (influencia interna). Además, atendiendo a los diferentes niveles de interacción social, se consideró que la influencia interna puede responder a un efecto de imitación cuando se considera la cantidad total de adoptadores en el sistema (nivel macro), o a un efecto de externalidad positiva cuando se considera la cantidad de vecinos adoptadores para cada individuo (nivel micro).

Nótese que el umbral de adopción se modeló de manera estocástica a lo largo de la población y el tiempo, y que los pesos de cada una de las influencias se consideraron iguales para los individuos. Sin embargo, así como en las reglas anteriores, las estocasticidad viene dada por la posición de cada individuo dentro de la red social.

R4: Regla sociológica: esta regla se implementó con base en la TPB, y se presenta en la Figura 4. 


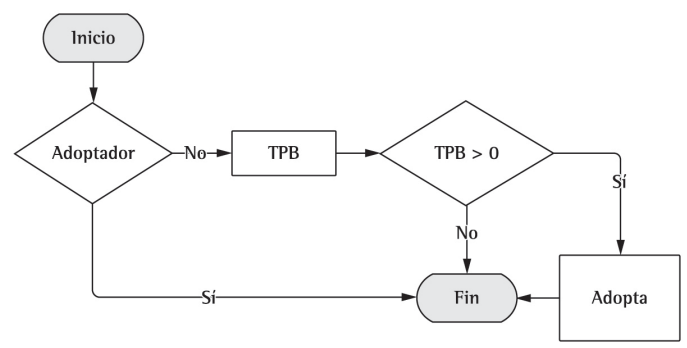

Figura 4. Regla sociológica. Fuente: los autores.

En cada paso de simulación, los individuos no adoptadores calculan el valor de la regla de decisión TPB. Si este valor es positivo, el individuo se convierte en adoptador.

Atendiendo al trabajo presentado por Zhang et al. (2011), la actitud hacia la adopción de cada individuo se consideró dependiente del precio de la innovación y de la publicidad recibida (estrategia de los proveedores), la norma subjetiva se modeló como una función de las influencias internas de la imitación y la externalidad positiva (de forma similar a como se hizo en la regla de Bass), y el control percibido se entendió como el resultado de la claridad de la regulación que rige a la innovación (acciones gubernamentales).

A fin de hacer esta regla altamente sofisticada para fines comparativos en la experimentación, se consideraron tantos umbrales como factores de la TPB, los cuales se modelaron de forma estocástica a lo largo de los individuos que conforman la red social. En este sentido, se consideró umbral para cada uno de los subfactores que componen el factor actitud (precio y publicidad), un umbral para la norma subjetiva (los subfactores son considerados insumos para el cálculo del nivel real de la norma, cuyos pesos se representaron determinísticos entre los individuos) y un nivel de reserva para el control percibido.

A diferencia de las demás reglas consideras que se basan únicamente en las dinámicas sociales, esta regla requiere especificar las características de la innovación relativas al precio, la intensidad de la publicidad o información disponible sobre la innovación y la claridad de la regulación que provee el sector gubernamental al respecto de la innovación, que constituirán los niveles reales de cada factor.

En términos generales, le cálculo de del valor de la regla TPB implica comparar los umbrales contra los valores reales de cada variable, arrojando una medición de cuán favorable o desfavorable resulta cada factor en la decisión de adopción; dichas medidas de favorabilidad se ponderan en función de los pesos de cada factor, para arrojar un valor de adopción, como se presenta en la Ecuación 2.
$T P B_{i t}=W p r_{i} \times V p r_{i t}+W p b_{i} \times V p b_{i t}+$

$W n s_{i} \times V n s_{i t}+W c t_{i} \times V c t_{i t}$

Donde:

- $\mathrm{TPB}_{\mathrm{it}}=$ valor de la regla TPB para el individuo $i$ en el momento $t$.

- $\mathrm{Wpr}_{\mathrm{i}}=$ peso que da el individuo $i$ a la variable precio en su decisión de adopción.

- $\mathrm{Wpb}_{\mathrm{i}}=$ peso que da el individuo $i$ a la variable publicidad en su decisión de adopción.

- $\mathrm{Wns}_{\mathrm{i}}=$ peso que da el individuo $i$ a la variable norma subjetiva en su decisión de adopción.

- $\mathrm{Wct}_{\mathrm{i}}=$ peso que da el individuo $i$ a la variable control en su decisión de adopción.

- $\mathrm{Vpr}_{\mathrm{i}}=$ medida relativa de la diferencia entre precio de la innovación y el precio umbral que tiene el individuo $i$

- $\mathrm{Vpb}_{\mathrm{it}}=$ medida relativa de la diferencia entre la publicidad de la innovación en el momento $t$ y la publicidad umbral que tiene el individuo $i$.

- $\mathrm{Vns}_{\mathrm{it}}=$ medida relativa de la diferencia entre la presión social (norma subjetiva) que es ejercida al individuo $i$ en el momento $t$, y el la presión social umbral que tiene el individuo $i$.

- $\mathrm{Vct}_{\mathrm{i}}=$ medida relativa de la diferencia entre el control de la innovación y el control umbral que tiene el individuo $i$.

La suma de los pesos debe corresponder a la unidad, como lo expresa la Ecuación 3.

$W p r_{i}+W p b_{i}+W n s_{i}+W c t_{i}=1$

Las Ecuaciones 4, 5, 6 y 7 expresan la manera en que se calcularon las distancias relativas para cada uno de los conceptos asociados.

$$
\begin{aligned}
& V p r_{i}=\frac{P r U_{i}-P r}{\operatorname{Pr} U_{i}} \\
& V p b_{i t}=\frac{P b_{t}-P b U_{i}}{P b U_{i}} \\
& V n s_{i t}=\frac{N s_{i t}-N s U_{i}}{N s U_{i}} \\
& V c r_{i}=\frac{C r-C r U_{i}}{C r U_{i}}
\end{aligned}
$$

Donde:

- $\operatorname{PrU}_{\mathrm{i}}=$ precio umbral del individuo $i$.

- $\operatorname{Pr}=$ precio de la innovación.

- $\mathrm{Pb}_{\mathrm{t}}=$ publicidad de la innovación en el momento $t$

- $\mathrm{PbU}_{\mathrm{i}}=$ publicidad umbral del individuo $i$. 
- $\mathrm{Ns}_{\mathrm{it}}=$ la presión social (norma subjetiva) que es ejercida al individuo $i$ en el momento $t$

- $\mathrm{NsU}_{\mathrm{i}}=$ presión social umbral del individuo $i$.

- $\mathrm{Cr}$ = medida del control de la innovación.

- $\mathrm{CrU}_{\mathrm{i}}=$ control umbral del individuo $i$.

Las medidas de diferencias relativas pueden ser negativas (en cuyo caso afectan negativamente la decisión de adopción) o positivas (en cuyo caso favorecen la decisión de adopción).

Nótese, además, que existe una correlación negativa entre la variable precio y la intención de adopción (a mayor precio, menor intención de adopción), mientras que la correlación es positiva para las variables publicidad, norma subjetiva y control.

El cálculo de la presión social de cada individuo en cada momento dependerá de la cantidad de adoptadores en toda la red (efecto imitación) y de adoptadores inmediatos en su vecindario (efecto externalidad), de manera similar al funcionamiento de la regla de Bass pero sin considerar la propensión a la adopción, la cual se asume recogida en los umbrales de la regla TPB. La Ecuación 8 presenta este cálculo.

$N s_{i t}=\frac{I \times \frac{A_{t}}{N}+E \times \frac{V A_{i t}}{V_{i}}}{2}$

Donde:

- 1 = coeficiente de imitación.

- $\mathrm{A}_{\mathrm{t}}=$ cantidad de adoptadores que hay en la red en el momento $t$.

- $\mathrm{N}=$ total de individuos en la red

- $\mathrm{E}=$ coeficiente de externalidad

- $\mathrm{VA}_{\mathrm{it}}=$ cantidad de vecinos adoptadores al individuo $i$ en el momento $t$.
- $\mathrm{V}_{\mathrm{i}}=$ cantidad de vecinos al individuo $i$.

Finalmente, la Tabla 1 presenta las diferentes reglas consideradas atendiendo a su nivel de complejidad, así como las variables que intervienen en cada una de ellas y la forma como fueron implementadas en el modelo.

\section{Experimentación}

Con el objetivo de evaluar el impacto de la regla de decisión en la difusión de una innovación se diseñaron 4 experimentos, cada uno de los cuales corresponde a la simulación de la difusión bajo cada una de las reglas de decisión consideradas. Dados los elementos estocásticos involucrados en las reglas de decisión, en cada uno de los experimentos se llevaron a cabo 1000 simulaciones, que tuvieron lugar a lo largo de una red estática tipo mundo pequeño compuesta por 500 nodos. La Tabla 2 presenta las características de cada uno de los experimentos conducidos.

El proceso llevado a cabo para el análisis experimental se presenta en la Figura 5.

El proceso comienza con la elección de una de las reglas de interés, que configura un experimento $i$. Acto seguido viene la determinación de los valores de los parámetros que caracterizarán el experimento, y su respectiva representación en el modelo de simulación. El modelo se ejecuta para obtener la simulación con las condiciones deseadas, un proceso que se realiza 1000 veces para cada experimento atendiendo a los elementos estocásticos incluidos en las reglas de decisión; el resultado de cada simulación es la cantidad de adoptadores en cada uno de los pasos de simulación. Finalmente, los datos arrojadas por las simulaciones son procesados para extraer las estadísticas de interés, y se procede con su análisis

Tabla 1. Reglas de decisión consideradas.

\begin{tabular}{|c|c|}
\hline Regla de decisión & Variable \\
\hline 1. Umbrales con externalidades positivas & $\begin{array}{l}\text { - Porcentaje de innovadores (variable de entrada) } \\
\text { - Umbral de adopción (u: determinístico) }\end{array}$ \\
\hline 2. Umbrales con externalidades positivas y negativas & $\begin{array}{l}\text { - Porcentaje de innovadores (variable de entrada) } \\
\text { - Umbral de adopción (u: determinístico) } \\
\text { - Umbral de abandono (u: determinístico) }\end{array}$ \\
\hline 3. Bass & $\begin{array}{l}\text { - Umbral de adopción (u: uniforme) } \\
\text { - Influencia externa - publicidad (p: determinístico) } \\
\text { - Influencia interna: } \\
\text { - Imitación (p: determinístico) } \\
\text { - Extrenalidad (p: determinístico) } \\
\end{array}$ \\
\hline 4. Theory of Planned Behavior (TPB) & $\begin{array}{l}\text { - Actitud } \\
\text { - Precio (u: normal. s: uniforme) } \\
\text { - Publicidad (u: uniforme. s: uniforme) } \\
\text { - Norma subjetiva (u: uniforme. s: uniforme) } \\
\text { - Imitación (p: determinístico) } \\
\text { - Externalidad (p: determinístico) } \\
\text { - Regulación (u: uniforme. s: uniforme) }\end{array}$ \\
\hline
\end{tabular}

Notación --> u: umbral. p: peso. s: sensibilidad. Fuente: los autores. 
Tabla 2. Características de los experimentos conducidos.

\begin{tabular}{|c|c|c|c|c|}
\hline Regla de decisión & Variable & \multicolumn{3}{|c|}{ Valor } \\
\hline \multirow{2}{*}{$\begin{array}{l}\text { 1. Umbrales con externalidades } \\
\text { positivas }\end{array}$} & Porcentaje de innovadores & \multicolumn{3}{|c|}{$5 \%$} \\
\hline & Umbral de adopción & \multicolumn{3}{|c|}{$25 \%$} \\
\hline \multirow{3}{*}{$\begin{array}{l}\text { 2. Umbrales con externalidades } \\
\text { positivas y negativas }\end{array}$} & Porcentaje de innovadores & \multicolumn{3}{|c|}{$5 \%$} \\
\hline & Umbral de adopción & \multicolumn{3}{|c|}{$25 \%$} \\
\hline & Umbral de abandono & \multicolumn{3}{|c|}{$90 \%$} \\
\hline \multirow{4}{*}{ 3. Bass } & Umbral de adopción & \multicolumn{3}{|c|}{$\mathrm{U}[0,1]$} \\
\hline & Influencia externa - publicidad & \multicolumn{3}{|c|}{0,1} \\
\hline & Influencia interna - imitación & \multicolumn{3}{|c|}{0,5} \\
\hline & Influencia interna - wxtrenalidad & \multicolumn{3}{|c|}{0,5} \\
\hline \multirow{7}{*}{ 4. Theory of Planned Behavior (TPB) } & & Reserva & Sesibilidad & Innovación \\
\hline & Actitud - precio umbral & $\mathrm{N}(120,2)$ & $\mathrm{U}[0,1]$ & 100 \\
\hline & Actitud - publicidad umbral & $\mathrm{U}[0,2,0,5]$ & $\mathrm{U}[0,1]$ & 0,25 \\
\hline & Norma subjetiva - umbral & $\mathrm{U}[0,5,1]$ & $\mathrm{U}[0,1]$ & NA \\
\hline & Norma subjetiva - imitación & 0,4 & NA & NA \\
\hline & Norma subjetiva - externalidad & 0,7 & NA & NA \\
\hline & Regulación & $\mathrm{N}(0,4,0,6)$ & $1-$ & 0,5 \\
\hline
\end{tabular}

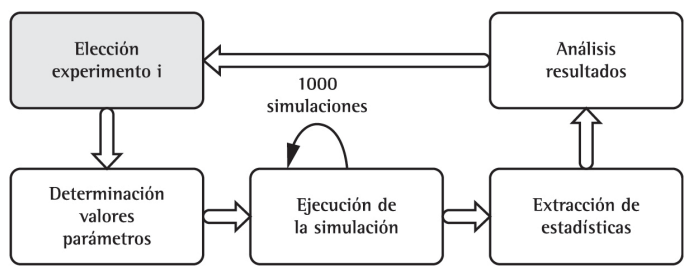

Figura 5. Proceso de análisis experimental. Fuente: los autores.

antes de continuar con la elección de otro experimento. De esta manera se realiza sucesivamente hasta haber abarcado todos los experimentos de interés.

\section{Resultados y discusión}

La Figura 6 presenta los resultados de las diferentes trayectorias de difusión para las diferentes reglas usadas en el experimento. Atendiendo a la estocasticidad presente en el fenómeno, las gráficas presentan las curvas de los valores máximos y mínimos obtenidos en las simulaciones, así como la trayectoria de difusión media correspondiente a cada regla (cantidad de adoptadores acumulados en el tiempo) y la dispersión promedio de las distintas simulaciones en cada caso, entendida como una medición global de qué tan alejadas se encuentran las trayectorias de difusión entre sí para una misma regla de decisión.

De acuerdo con los resultados obtenidos, las reglas Bass y umbrales con externalidades positivas y negativas arrojan la mayor dispersión promedio, mientras que las reglas umbrales con externalidades positivas y TPB tienen una dispersión menor. Sorprende el hecho de que la regla TPB presente una baja dispersión entre sus simulaciones, considerando que es la regla que incluye una mayor cantidad de variables aleatorias; esto puede deberse a la parametrización que se hizo en el experimento de las distribuciones de las variables aleatorias correspondientes a esta regla, o a un efecto anulador entre las variaciones que se computa al generar los resultados de difusión acumulados.

La Figura 7 presenta las trayectorias de difusión media correspondientes a las diferentes reglas de decisión en el experimento, así como la cantidad media de nuevos adoptadores.

Las trayectorias de difusión correspondientes a las reglas de Bass y TPB tienen un comportamiento similar a la forma en "S" que se espera en las trayectorias de difusión de una innovación, mientras que las reglas umbrales con externalidades positivas y umbrales con externalidades positivas y negativas arrojan una curva que no se asemeja a esta forma sino que presenta un comportamiento creciente asintótico con un tope de adoptadores.

Este fenómeno se observa también en las curvas de nuevos adoptadores, en las cuales las reglas de Bass y TPB presentan el comportamiento descrito por Rogers (1983), mientras que las demás exhiben un decrecimiento exponencial en el número de nuevos adoptadores. La regla umbrales con externalidades positivas y negativas es la única que presenta decrecimientos en la curva de difusión acumulada, lo cual responde al hecho de ser la única regla diseñada para considerar des-adopciones durante el fenómeno de la difusión.

$\mathrm{Si}$ bien visualmente se observan diferencias entre las curvas de difusión obtenidas, con el fin de medir la semejanza en las curvas de difusión y siguiendo la metodología presentada por Rahmandad \& Sterman 

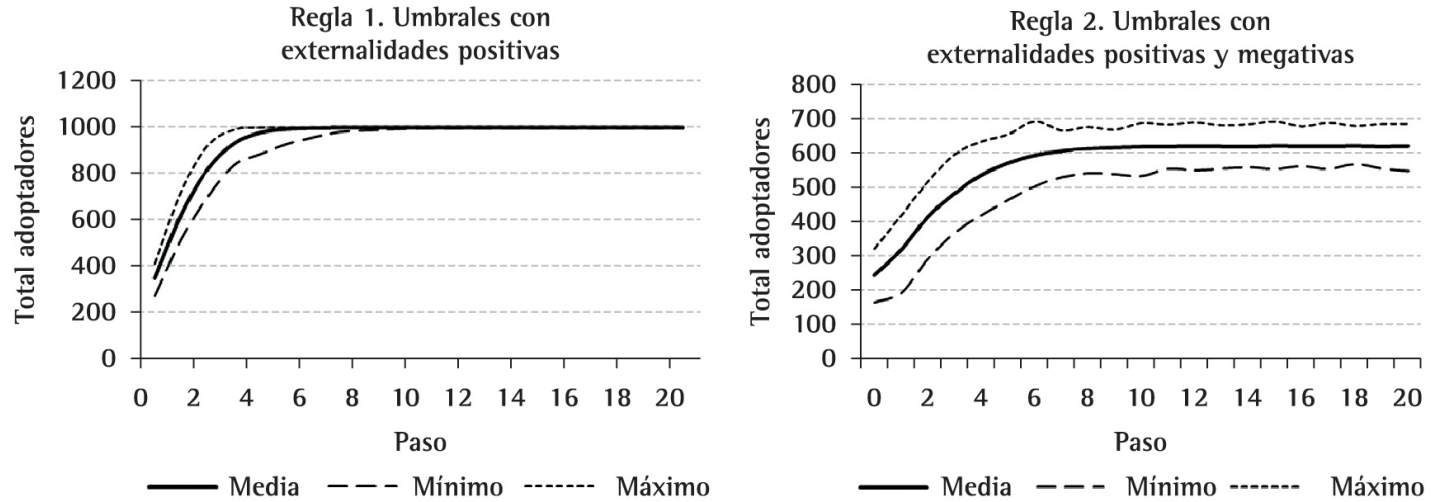

Regla 3. Bass
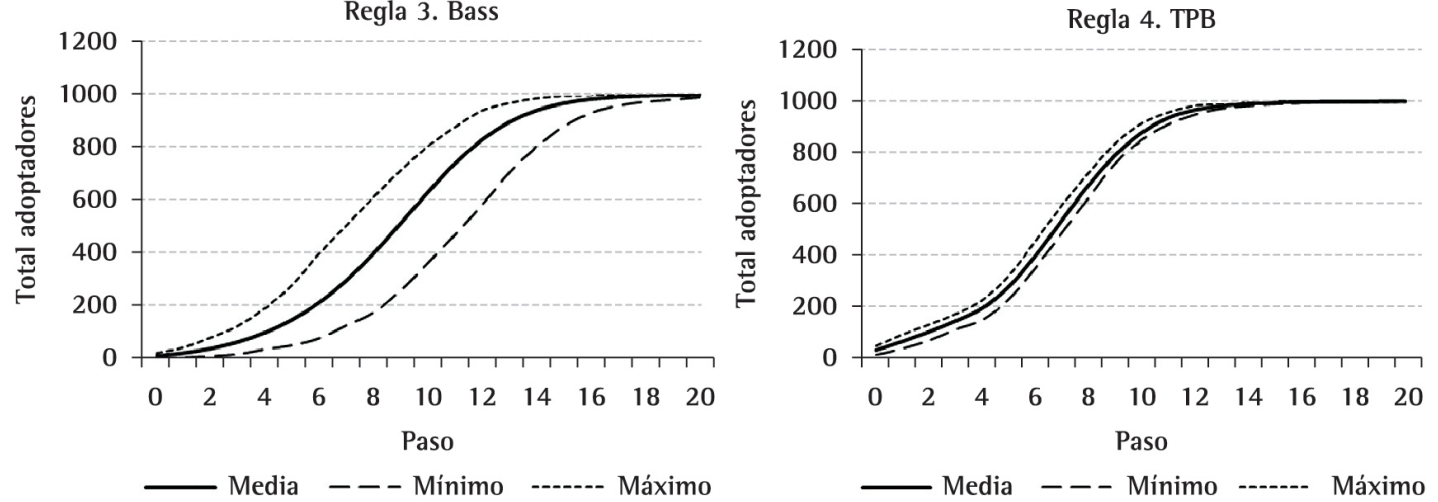

Figura 6. Trayectorias de difusión y coeficiente de dispersión. Fuente: los autores.

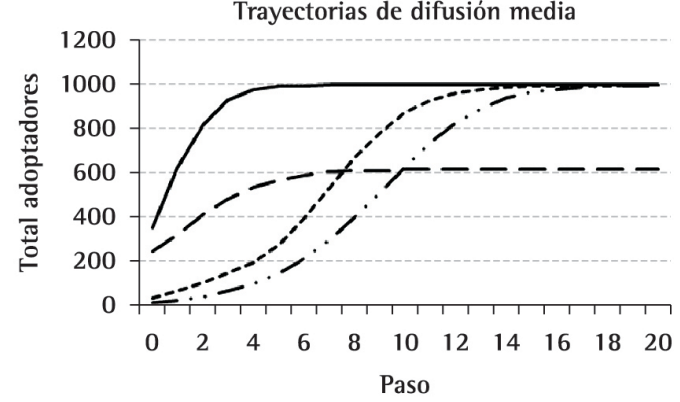

$-\mathrm{R} 1$. Umbrales con externalidades positivas

- R2. Umbrales con externalidades positivas y negativas

$-\cdots$ R3. Bass

---- R4. TPB

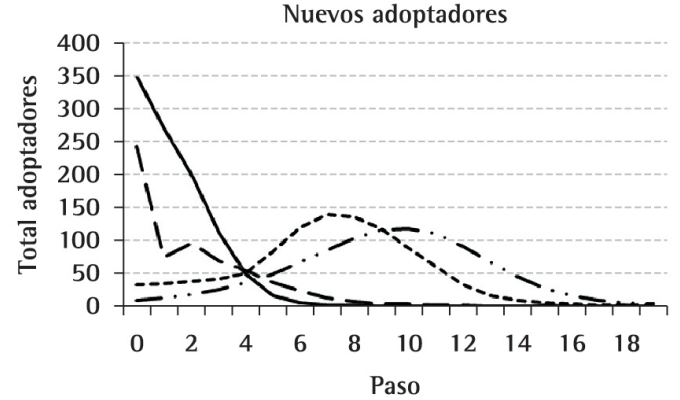

R1. Umbrales con externalidades positivas

- - R2. Umbrales con externalidades positivas y negativas - $\cdots$ R3. Bass

-----R4. Theory of Planned Behavior (TPB)

Figura 7. Trayectorias de difusión media de las reglas de difusión. Fuente: los autores.

(2008), para cada uno de los experimentos se tomaron tres mediciones o estadísticos representativos respecto a la cantidad de adoptadores nuevos que da origen a las curvas de difusión: cantidad máxima de nuevos adoptadores (Amáx), tiempo que transcurre entre la primera adopción y la cantidad máxima de nuevos adoptadores (Tmáx) y la fracción de la población potencial que, finalmente, adopta la innovación $(F)$.
La Tabla 3 presenta el valor medio y la deviación estándar de dichos estadísticos para cada condición del experimento.

La cantidad máxima de nuevos adoptadores bajo la regla umbrales con externalidades positivas es 349 , la cual se obtiene en el primer instante en que la difusión tiene lugar, al igual que ocurre con la regla umbrales con externalidades positivas y negativas, 
cuya cantidad máxima de nuevos adoptadores es 242 . Las reglas Bass y TPB obtienen la cantidad máxima de nuevos adoptadores en los períodos 11 y 8 , siendo dichos valores 126 y 144, respectivamente.

Asimismo, las reglas analizadas en el experimento generan adopciones en casi el 100\% de la población, excepto en el caso de la regla de umbrales con externalidades positivas y negativas, cuyo porcentaje de adoptadores finales se encuentra alrededor del 65\% de la población potencial de adoptadores gracias al desestímulo que genera la saturación del mercado en este escenario.

Para evaluar la igualdad o no de estos estadísticos, se diseñó una prueba de hipótesis para la diferencia de medias con muestras grandes obtenidas a partir de poblaciones con distribuciones desconocidas y varianzas poblacionales desconocidas, como se presenta a continuación:

$$
\begin{gathered}
H_{o}: \mu_{i}=\mu_{j} \\
H_{a}: \mu_{i} \neq \mu_{j} \\
\forall i, j=1,2,3,4 \quad i \neq j
\end{gathered}
$$

Estadístico de prueba para cada par $(\mathrm{i}, \mathrm{j})$ seleccionado: $Z=\frac{\bar{x}_{i}-\bar{x}_{j}}{\sqrt{\frac{S_{i}^{2}}{n_{i}}-\frac{S_{j}^{2}}{n_{j}}}} \sim N(0,1)$

La Tabla 4 presenta el valor del estadístico para cada uno de los posibles pares de curvas, en cada una de las tres variables de interés.

Las diferencias en las mediciones Amáx, Tmáx y $F$ entre los experimentos resultan estadísticamente significativas $(p<0,001)$ en todos los posibles pares de experimentos, hecho frecuente cuando se realizan simulaciones a gran escala (en este caso 1000 simulaciones para cada experimento).

Es decir, que puede afirmarse que la cantidad máxima de nuevos adoptadores, el tiempo que transcurre entre la primera adopción y la cantidad máxima de nuevos adoptadores, y la fracción de la población que adopta la innovación, cambian al cambiar la regla con la que se modela el proceso de decisión de los individuos. Esto indica que la regla de decisión tiene un impacto significativo en

Tabla 3. Estadísticos de las curvas de difusión.

\begin{tabular}{lcccc}
\hline & $\begin{array}{c}\text { R1. Umbrales con } \\
\text { externalidades positivas }\end{array}$ & $\begin{array}{c}\text { R2. Umbrales con externalidades } \\
\text { positivas y negativas }\end{array}$ & R3. Bass & $\begin{array}{c}\text { R4. Theory of Planned } \\
\text { Behavior (TPB) }\end{array}$ \\
\hline Amax & 349 & 242 & 126 & 144 \\
Media & 20 & 25 & 8 & 8 \\
$\quad$ Desvest & 1 & & & 8 \\
Tmax & 0 & 1 & 11 & 1 \\
Media & & 0 & & 99,98 \\
Desvest & 100,00 & & 99,88 & 0,0 \\
F & 0,0 & 65,06 & 0,1 & \\
Media (\%) & & 1,1 & & \\
Desvest (\%) & & &
\end{tabular}

\begin{tabular}{|c|c|c|c|c|c|c|c|c|c|}
\hline & \multicolumn{3}{|c|}{$\begin{array}{l}\text { R2. Umbrales con externalidades } \\
\text { positivas y negativas }\end{array}$} & \multicolumn{3}{|c|}{ R3. Bass } & \multicolumn{3}{|c|}{$\begin{array}{l}\text { R4. Theory of Planned Behavior } \\
\text { (TPB) }\end{array}$} \\
\hline & Amax & $\operatorname{Tmax}$ & $F$ & Amax & $\operatorname{Tmax}$ & $F$ & Amax & Tmax & $F$ \\
\hline $\begin{array}{l}\text { R1. Umbrales con } \\
\text { externalidades positivas }\end{array}$ & 104,9 & NA & 1041 & 328 & -351 & 29 & 299 & -369 & 14 \\
\hline $\begin{array}{l}\text { R2. Umbrales con } \\
\text { externalidades positivas y } \\
\text { negativas }\end{array}$ & & & & 138,8 & -318 & -1030 & 117 & -369 & -1040 \\
\hline R3. Bass & & & & & & & -51 & 66 & -23 \\
\hline
\end{tabular}

Fuente: los autores.

Tabla 4. Estadísticos z para prueba de igualdad de medias.

Fuente: los autores.

Tabla 5. Estadísticos chi-cuadrado para prueba de bondad de ajuste.

\begin{tabular}{lcc} 
& $\begin{array}{c}\text { R2. Umbrales con externalidades } \\
\text { positivas y negativas }\end{array}$ & $\begin{array}{c}\text { R4. Theory of Planned } \\
\text { Behavior (TPB) }\end{array}$ \\
\hline R1. Umbrales con externalidades positivas & 3081 & 5968 \\
R2. Umbrales con externalidades positivas y negativas & & 3981 \\
R3. Bass & 3423 & 1529 \\
\hline
\end{tabular}

Fuente: los autores. 
la trayectoria de difusión simulada en los modelos a nivel individual de difusión de innovaciones, de manera que diferentes reglas generan diferentes curvas de difusión.

Estos estadísticos, sin embargo, corresponden a mediciones puntuales sobre las series de datos, dejando por fuera los demás puntos de la misma que
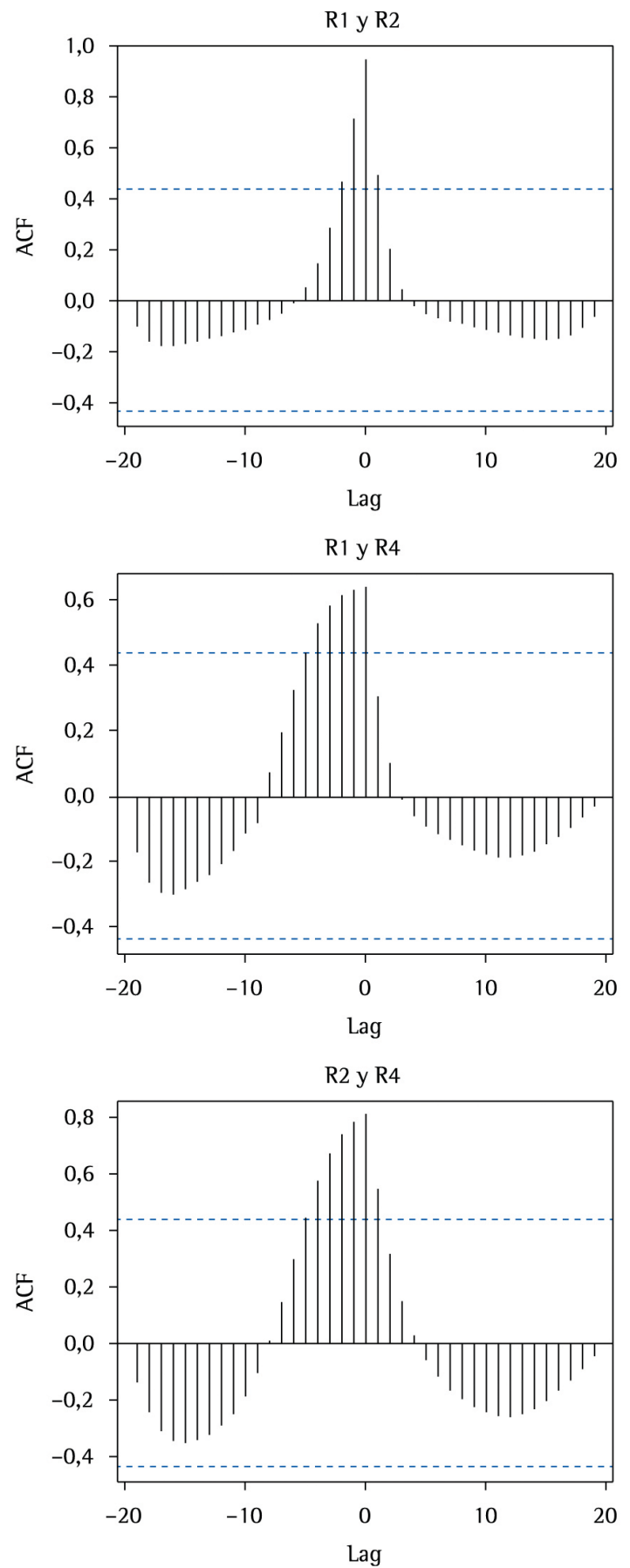

podrían ser coincidentes entre las series. Siguiendo el procedimiento presentado por Pearson (Plackett, 1983), se realizó una prueba de bondad de ajuste basada en el estadístico chi-cuadrado para cada uno de los pares posibles de trayectorias de difusión, con el fin de probar la hipótesis de igualdad entre los pares de trayectorias seleccionados, así:
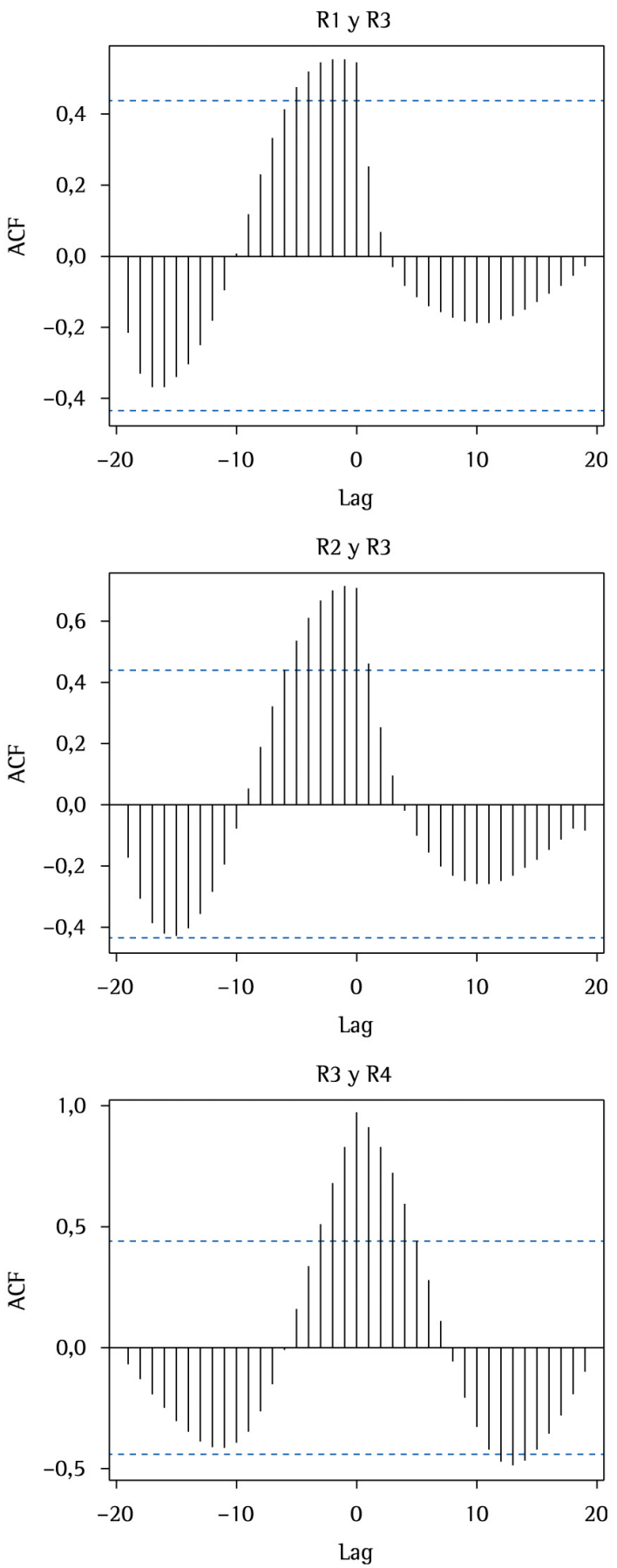

Figura 8. Correlación cruzada. Fuente: los autores - paquete estadístico $\mathrm{R}^{\circledR}$. 


$$
\begin{gathered}
H_{o}: C_{i}=C_{j} \\
H_{a}: C_{i} \neq C_{j} \\
\forall i, j=1,2,3,4 \quad i \neq j
\end{gathered}
$$

Estadístico de prueba para cada par $(\mathrm{i}, \mathrm{j})$ seleccionado: $\chi_{n-k-1}^{2}=\sum_{k=1}^{n} \frac{\left(D_{i k}-D_{j k}\right)^{2}}{D_{j k}}$ Donde:

- $\mathrm{D}_{\mathrm{i} \mathrm{k}}$ : dato $\mathrm{k}$ de la serie $i$.

- $n-k-1$ : grados de libertada de la distribución chi-cuadrado (en el caso particular, $n=20$ pasos de simulación, y $k=1$ dado que se utilizó la el valor medio en cada paso considerando el total de simulaciones realizadas).

De esta manera, se realizaron 6 pruebas de bondad de ajuste, cuyo valor del estadístico chi-cuadrado se presenta en la Tabla 5.

Atendiendo a que el valor $\chi_{20}^{2}-1-1$ para un valor $p$ de 0,01 es 34,81 , en todos los casos analizados se rechaza Ho, de manera que, con una confianza del 99\%, puede afirmarse que no existe evidencia suficiente para concluir que las curvas de difusión son iguales entre sí.

Si bien los estadísticos indican que las trayectorias de difusión resultan diferentes cuando cambia la regla de decisión, puede observarse una similitud en el comportamiento de las curvas de difusión entre las reglas de umbrales, y entre las reglas Bass y TPB. Con el objetivo de chequear la semejanza en la forma de las diferentes curvas de difusión, la Figura 8 presenta las gráficas de la correlación cruzada entre los pares de curvas de difusión posibles en el experimento.

Se obtienen correlaciones cercanas a 1 entre las curvas de difusión de las reglas umbrales con externalidades positivas y umbrales con externalidades positivas y negativas, así como de las reglas Bass y $\mathrm{TPB}$, en ambos casos sin rezago. Atendiendo a que las curvas de difusión son estadísticamente distintas, esta correlación indica que las curvas tienen formas similares entre si si bien no conservan la escala.

\section{Conclusiones}

Este artículo presentó los resultados de un experimento que compara el efecto que diferentes reglas de decisión tienen en el patrón de difusión de una innovación usando un modelo a nivel individual basado en agentes, suponiendo una estructura de red con características de mundo pequeño. Se consideraron una regla de decisión basada en umbrales con externalidades positivas, una regla de decisión basada en umbrales que incluye externalidades positivas y negativas, una regla de decisión que replica los conceptos presentados por Bass y una regla de decisión basada en la Teoría del Comportamiento Planeado.

Los estadísticos más relevantes en las trayectorias de difusión obtenidos indican que las curvas de difusión son estadísticamente diferentes para las distintas reglas del experimento, un resultado similar al obtenido al probar la hipótesis de igualdad entre las curvas de difusión, la cual fue rechazada. Además, las curvas de difusión que se producen con las reglas umbrales con externalidades positivas y umbrales con externalidades positivas y negativas presentan una alta correlación en el rezago cero, de la misma manera como sucede con las reglas Bass y TPB, lo cual indica que las curvas tienen formas similares entre sí para cada par mencionado, si bien no conservan la escala.

De esta manera, puede afirmarse que la regla de decisión empleada en el modelado del proceso de adopción de los individuos dentro de los modelos de difusión a nivel individual, tiene un impacto significativo en la trayectoria de difusión que pronostican dichos modelos. Este hecho resalta para los investigadores del fenómeno de la difusión de innovaciones la importancia de diseñar, calibrar y probar adecuadamente la regla de decisión de adopción considerada en sus modelos, a fin de obtener resultados cercanos a la realidad.

\section{Trabajo futuro}

Investigaciones que consideren el efecto de la regla de decisión en redes aún más cercanas a las redes sociales del mundo real también son deseables; en este sentido, investigaciones que incluyan redes de difusión que cambian de manera endógena en el tiempo o que incluyan patrones de leyes de potencias podrian develar con mayor precisión el efecto de la regla de decisión en las curvas de difusión pronosticados por los modelos a nivel individual.

Los resultados de la correlación cruzada abren una puerta de investigación, al sugerir la posibilidad de que unas reglas sean recogidas por otras. En este sentido, investigaciones encaminadas a la construcción de modelos de adopción robustos, capaces de soportar diferentes lógicas de razonamiento y de predecir diferentes patrones de difusión dentro de una misma representación, enriquecerían el modelado del fenómeno de la difusión de las innovaciones.

El hecho de que los resultados de esta investigación muestren que la regla de decisión tiene un impacto importante en los pronósticos hechos por los modelos de difusión, indica que son necesarios más y mejores mecanismos para validar las reglas de decisión de los individuos en el mundo real, previo a su modelado. Aunque algunas investigaciones recientes hacen 
uso de entrevistas con expertos y encuestas con los potenciales consumidores para tal fin, sorprende el hecho de que no se haya desarrollado ni validado metodología alguna para llevar a cabo este tipo de investigaciones. En esta misma dirección, es necesario que los estudios de aplicación que hacen uso de modelos de difusión de innovaciones a nivel individual presenten sus resultados basados en una técnica confiable para la validación de la regla de decisión empleada, o bien, consideren diferentes posibles reglas y presenten los distintos escenarios de difusión en cada caso.

\section{Referencias}

Ajzen, 1. (1985). From intentions to actions: a theory of planned behavior. In J. Kuhl \& J. Beckemann (Eds.), Action control: from cognition to behavior ( $p p$. 11-39). New York: Springer-Verlag. http://dx.doi. org/10.1007/978-3-642-69746-3_2

Alkemade, F., \& Castaldi, C. (2005). Strategies for the diffusion of innovations on social networks. Computational Economics, 25(1-2), 3-23. http://dx.doi.org/10.1007/ s10614-005-6245-1

Allen, B. (1982). Some stochastic processes of interdependent demand and technological diffusion of an innovation exhibiting externalities among adopters. International Economic Review, 23(3), 595-608. http://dx.doi. org $/ 10.2307 / 2526377$

Bagozzi, R. P. (2007). The legacy of the technology acceptance model and a proposal for a paradigm shift. Journal of the Association for Information Systems, 8(4), 244-254.

Barabási, A.-L. (1999a). Mean-field theory for scale-free random netowork. Physica A: Statistical Mechanics and its Applications, 272(1-2), 173-187. http://dx.doi. org/10.1016/S0378-4371(99)00291-5

Barabási, A.-L. (1999b). Emergence of scaling in random networks. Science, 286(5439), 509-512. PMid:10521342. http://dx.doi.org/10.1126/science.286.5439.509

Bass, F. M. (1969). A new product gowth for model consumer durables. Management Science, 15(5), 215-227. http:// dx.doi.org/10.1287/mnsc. 15.5.215

Berger, T. (2001). Agent-based spatial models applied to agriculture: a simulation tool for technology diffusion, resource use changes and policy analysis. Agricultural Economics, 25(2-3), 245-260. http://dx.doi. org/10.1111/j.1574-0862.2001.tb00205.x

Buchanan, J. M. (1969). External diseconomies, corrective taxes, and market structure. The American Economic Review, 59(1), 174-177.

Chuttur, M. (2009). Overview of the technology acceptance model: origins, developments and future directions. Sprouts: Working Papers on Information Systems, 9(37), 1-23.

Gardner, M. (1970). Mathematical games: the fantastic combinations of John Conway's new solitaire game "Life". Scientific American, 223(4), 120-123. http:// dx.doi.org/10.1038/scientificamerican 1070-120

Georgescu, S., \& Okuda, H. (2008). A distributed multi-agent framework for simulating the diffusion of innovations.
Journal of Power and Energy Systems, 2(6), 1320-1332. http://dx.doi.org/10.1299/jpes.2.1320

Gilbert, N. (1997). A simulation of the structure of academic science. Sociological Research Online, 2(2), 1-17. http:// dx.doi.org/10.5153/sro.85

Goldenberg, J., Libai, B., Solomonc, S., Jand, N., \& Stauffere, D. (2000). Marketing percolation. Physica A: Statistical Mechanics and its Applications, 284(1-4), 335-347. http://dx.doi.org/10.1016/S0378-4371(00)00260-0

Goldenberg, J., \& Efroni, S. (2001). Using cellular automata modeling of the emergence of innovations. Technological Forecasting and Social Change, 68(3), 293-308. http:// dx.doi.org/10.1016/S0040-1625(00)00095-0

Hale, J., Householder, B., \& Green, K. (2002). The theory of reasoned action. In J. Dillard \& M. Pfau (Eds.), The persuasion handbook: developments in theory and practice (pp. 865). Thousand Oaks: Sage Publications.

Hsu, C. L., \& Lin, J. C. (2008). Acceptance of blog usage: the roles of technology acceptance, social influence and knowledge sharing motivation. Information \& Management, 45(1), 65-74. http://dx.doi.org/10.1016/j. im.2007.11.001

Katz, M. L., \& Shapiro, C. (1986). Technology adoption in the presence of network externalities. The Journal of Political Economy, 94(4), 822-841. http://dx.doi. $\operatorname{org} / 10.1086 / 261409$

Katz, M. L., \& Shapiro, C. (1992). Product introduction with network externalities. The Journal of Industrial Economics, 40(1), 55-83. http://dx.doi. org/10.2307/2950627

Kemp, R., \& Volpi, M. (2008). The diffusion of clean technologies: a review with suggestions for future diffusion analysis. Journal of Cleaner Production, 16(1), S14-S21. http://dx.doi.org/10.1016/j.jclepro.2007.10.019

Kiesling, E., Günther, M., Stummer, C., \& Wakolbinger, L. M. (2012). Agent-based simulation of innovation diffusion: a review. Central European Journal of Operations Research, 20(2), 183-230. http://dx.doi.org/10.1007/ s10100-011-0210-y

Macvaugh, J., \& Schiavone, F. (2010). Limits to the diffusion of innovation: a literature review and integrative model. European Journal of Innovation Management, 13(2), 197221. http://dx.doi.org/10.1108/14601061011040258

Mahajan, V., Muller, E., \& Bass, F. (1990). New product diffusion models in marketing: a review and directions for research. The Journal of Marketing, 54(1), 1-26. http://dx.doi.org/10.2307/1252170

Manson, S. M. (2006). Bounded rationality in agentbased models: experiments with evolutionary programs. International Journal of Geographical Information Science, 20(9), 991-1012. http://dx.doi. org/10.1080/13658810600830566

Montalvo, C., \& Kemp, R. (2008). Cleaner technology diffusion: case studies, modeling and policy. Journal of Cleaner Production, 16(1), S1-S6. http://dx.doi. org/10.1016/j.jclepro.2007.10.014

Peres, R., Muller, E., \& Mahajan, V. (2010). Innovation diffusion and new product growth models: a critical review and research directions. International Journal of Research in Marketing, 27(2), 91-106. http://dx.doi. org/10.1016/j.ijresmar.2009.12.012 
Plackett, R. (1983). Karl Pearson and the chi-squared test. International Statistical Review, 51(1), 59-72. http:// dx.doi.org/10.2307/1402731

Rahmandad, H., \& Sterman, J. (2008). Heterogeneity and network structure in the dynamics of diffusion: comparing agent-based and differential equation models. Management Science, 54(5), 998-1014. http://dx.doi. org/10.1287/mnsc. 1070.0787

Rao, K. U., \& Kishore, V. V. N. (2010). A review of technology diffusion models with special reference to renewable energy technologies. Renewable and Sustainable Energy Reviews, 14(3), 1070-1078. http://dx.doi.org/10.1016/j. rser.2009.11.007

Rogers, E. M. (1983). Diffusion of innovations (Vol. 11, 3rd ed.). London: Collier Macmillan Publishers.

Rohlfs, J. (2001). Bandwagon effects in high-technology industries (Vol. 27). Cambridge: MIT Press.

Schelling, T. C. (1978). Micromotives and macrobehavior. New York: W. W. Norton \& Company.
Varian, H. R. (1985). Price discrimination and social welfare. The American Economic Review, 75(4), 870-875.

Venkatesh, V., \& Bala, H. (2008). Technology acceptance model 3 and a research agenda on interventions. Decision Sciences, 39(2), 273-315. http://dx.doi.org/10.1111/ j.1540-5915.2008.00192.x

Watts, D. J. (2000). Small worlds: the dynamics of networks between order and randomn. Mathematical Association of America, 1077), 664-668.

Watts, D. J., \& Strogatz, S. H. (1998). Models of the small world. Nature, 393, 440-442. PMid:9623998. http:// dx.doi.org/10.1038/30918

Zhang, T., Gensler, S., \& Garcia, R. (2011). A study of the diffusion of alternative fuel vehicles: an agent-based modeling approach. Journal of Product Innovation Management, 28(2), 152-168. http://dx.doi.org/10.1111/ j.1540-5885.2011.00789.x

\section{Impact of the decision rule in innovation diffusion modeling}

\section{Abstract}

In this, paper we analyze the impact of the decision rule to represent the behavior of individuals in the diffusion curve predicted by models of innovation diffusion at the individual level. We use an agent-based model, in which diffusion takes place in a small-world network, and analyze the phenomenon using 4 different decision rules: (1) threshold decision rule with positive externalities, (2) threshold decision rule with positive and negative externalities, (3) decision rule based on the Bass model and (4) decision rule based on the Theory of Planned Behavior. The results reject the equality hypothesis among different diffusion curves, so we conclude the decision rule has a significant impact on the diffusion curve predicted by diffusion models at the individual level.

\section{Keywords}

Simulation. Innovation diffusion. Adoption of innovations. Agent-based modeling. Decision rule. 